\title{
Causas de reprobación en ingeniería desde la perspectiva del académico y administradores
}

\author{
María Guadalupe Amado Moreno ${ }^{1}$, Ángel García Velázquez ${ }^{1}$, Reyna \\ Arcelia Brito Páez ${ }^{1}$, Bertha Ivonne Sánchez Luján² y Carlos Alfonso
}

Sagaste Bernal ${ }^{1}$

\section{Resumen}

La reprobación estudiantil es un problema añejo y complejo que tiene que irse monitoreando cada determinado tiempo en las instituciones de educación superior. El propósito de esta investigación fue identificar las causas de reprobación en las carreras de ingeniería del Instituto Tecnológico de Mexicali, en Baja California, México, desde la perspectiva de los actores educativos principales. En este documento se presentan los resultados obtenidos de los académicos y administradores. La información se recabó mediante una encuesta aplicada en febrero de 2013. El método utilizado es de tipo descriptivo-analítico. Pese a que los orígenes son variados, los académicos y administradores consideran que la principal causa de reprobación de los estudiantes de ingeniería es atribuible al rendimiento escolar del estudiante, es decir problemas relacionados a la falta de hábitos de estudio, de bases para cursar las asignaturas, dificultad para entender la asignatura y no asistir al aula o a las asesorías.

Palabras clave: Causas de reprobación, educación superior, perspectiva del académico.

2 Instituto Tecnológico de Cd. Jiménez, México. 


\begin{abstract}
Student reprobation is a age and complex problem that has to be monitoring by higher education institutions. The purpose of this research was to identify the failure causes of in engineering careers of the Instituto Tecnológico de Mexicali, Baja California, Mexico, from the perspective of the main educational actors. The results obtained from the academics and administrators are presented in this document. The information was collected through a survey conducted in February 2013. The method used is descriptiveanalytic. Although the origins are varied, academics and administrators consider that the main cause of disapproval of engineering students is attributable to school student performance, namely related to the lack of study habits, database problems to study subjects, difficulty understanding the subject and not attend classes or tutoring.
\end{abstract}

Key words: Causes of failure, higher education, academic perspective. 


\section{Introducción}

La reprobación en el nivel de educación superior tiene como consecuencia el rezago y la deserción. La disminución de los índices de reprobación estudiantil es tarea que demanda la participación de todos los actores involucrados de los distintos niveles de la comunidad educativa. Este difícil problema, requiere de un cambio en la forma en que los estudiantes visualizan su estadía en las instituciones de educación superior, la forma en que los docentes realizan su labor y una intervención inmediata de las autoridades educativas con acciones que garanticen la incorporación de elementos que favorezcan el análisis y prevención de las causas de reprobación, mediante la aplicación de mecanismos de control e instancias de toma de decisiones.

En los últimos años se ha detectado un aumento considerable en los índices de reprobación, debido a diferentes factores, que van desde el origen familiar, económico, hábitos de estudio, práctica docente, hasta causas administrativas. Es un problema que se agrava con el paso del tiempo, y esto a su vez puede provocar que los estudiantes abandonen sus estudios, lo que conlleva a pérdidas para las instituciones de educación superior (IES) y desánimo en los estudiantes (Durán Encalada y Díaz Hernández, 1990).

Este artículo es producto de la investigación "Análisis de las causas de reprobación en ingeniería en instituciones públicas", que se llevó a cabo en tres instituciones de educación superior pública en México: Instituto Tecnológico de Chihuahua, Instituto Tecnológico de Ciudad Jiménez e Instituto Tecnológico de Mexicali (ITM), el cual tuvo como objetivo identificar y analizar las causas de reprobación estudiantil en las carreras de ingeniería, desde la perspectiva del estudiante, académico y administradores.

Se exponen las causas de reprobación en ingeniería en el Instituto Tecnológico de Mexicali desde las perspectivas del académico y administradores, mientras que los resultados desde la visión del estudiante se encuentran reportados por Amado Moreno, García Velázquez, Sánchez Luján y Brito Páez (2013) en el V Congreso Internacional de Educación de la Universidad Autónoma de Baja California, México.

Cuatro de cada diez ingenieros se forma en los Institutos Tecnológicos, los cuales son IES pertenecientes a la Dirección General de Educación Superior Tecnológica en México (Sistema Nacional de Educación Superior Tecnológica, 2012) y sus estudiantes no son ajenos a esta problemática, y es en parte uno de los motivos para realizar esta investigación. 


\section{Antecedentes del problema}

La reprobación es un fenómeno que impacta en las Instituciones de Educación Superior, sobre todo en las carreras de ingeniería, y si bien es un problema añejo se considera necesario seguirlo estudiando para tener un acercamiento a la posible solución de este complejo problema.

Los resultados obtenidos por Nava Bustos, Rodríguez Roldán y Zambrano Guzmán en 2007, muestran que las causas de reprobación de los estudiantes del Centro Universitario de Ciencias de la Salud de la Universidad de Guadalajara, México se deben en primer lugar a problemas laborales y por empalme con el horario de clase, en segundo lugar a los problemas familiares y a dificultades en la materia, en tercer lugar.

Las materias asociadas a las ciencias básicas y sus aplicaciones han sido objeto de numerosos estudios. Morales Burgos, García Sosa y Escalante Triay en 2009, concluyen que la principal causa de reprobación en estudiantes de Mecánica de Fluidos de la Universidad Autónoma de Yucatán, México, está asociada a la falta de dedicación al estudio, ya que los estudiantes no asisten a las asesorías impartidas por sus profesores, ni realizan ejercicios extras a los revisados en clase. Como causa secundaria, los estudiantes mencionan la falta de planeación de sus actividades de estudio y organización académica.

Espinoza García (2005) clasifica las causas de la reprobación de la siguiente manera:

- Causas de origen social y familiar: desarticulación y/o disfuncionalidad familiar, desadaptación al medio por el origen sociocultural del que provienen, estudiantes que trabajan, problemas psicosociales y estudiantes casados y/o de paternidad o maternidad prematuras.

- Causas de origen psicológico: desubicación en propósitos de vida e inadecuada opción vocacional.

- Causas económicas: escasez de recursos y desempleo de los padres.

- Causas atribuibles al rendimiento escolar: perfiles de ingreso inadecuados y falta de hábitos de estudio.

- Causas fisicas: problemas de salud y alimentación inadecuada.

\section{Estudios de causas de reprobación en los Institutos Tecnológicos}

Mánica Zuccolotto, Cetina Canto y Pinzón Lizárraga (2008), presentan un estudio de las causas de reprobación en el Instituto Tecnológico de Mérida, basados en el desarrollo organizacional, en la que se analizan las aportaciones de estudiantes y profesores de la institución. Su objetivo fue el formular propuestas para disminuir el índice de reprobación a través de un diagnóstico a docentes y estudiantes; los 
resultados obtenidos giran en torno a grupos numerosos que dificultan la atención, falta de interés, compromiso y conocimientos previos en los estudiantes, aunado a factores administrativos como: asignación tardía de grupos, exigencias de llenado de formatos, constantes cambios en los programas y en algunos casos falta de equipo en laboratorios y talleres.

Los docentes del Instituto Tecnológico de Chihuahua II mencionan que el principal motivo de la reprobación de los estudiantes de la carrera industrial esta relacionado con el estudiante, sin embargo exponen que las estrategias didácticas utilizadas por el docente también impactan en la reprobación (González Montes, Rodríguez Galván, Treviso Burciaga, Flores Payán y Makita Aguilar, 2011).

Amado Moreno, García Velázquez, Sánchez Luján y Brito Páez (2013) reportan que desde la perspectiva del estudiante las causas de reprobación en el IT de Mexicali son: atribuibles al rendimiento escolar (falta de hábitos y motivación para el estudio, no tomar notas ni apuntes en clase y falta de conocimientos o bases para llevar las materias), a la institución (programas extensos, grupos numerosos y profesores que no dominan la materia) y al profesor (es muy exigente y no explica con claridad, ni domina la materia que imparte).

\section{Los Institutos Tecnológicos}

Los Institutos Tecnológicos (IT) son IES públicas que forman parte del Sistema Nacional de Institutos Tecnológicos (SNIT) en México. Está formado por 262 instituciones en todo el país, de los cuales 130 son institutos tecnológicos estatales o descentralizados y 126 federales, además, incorporado a este subsistema están 6 centros especializados: el Centro Nacional de Investigación y Desarrollo Tecnológico en Morelos, el Centro Interdisciplinario de Investigación y Docencia en Educación Técnica en Querétaro y cuatro Centros de Regionales de Optimización y Desarrollo de Equipo en Celaya, Orizaba, Mérida y Chihuahua (Sistema Nacional de Educación Superior Tecnológica, 2012).

La Dirección General de Educación Superior Tecnológica (DGEST) que engloba al SNIT actualmente trabaja con el Modelo Educativo para el Siglo XXI: Formación y desarrollo de competencias profesionales. El Modelo está dirigido a satisfacer la demanda de educación Superior Científica y Tecnológica en México (Vega Pérez, 2012) y está acorde a las exigencias internacionales sobre educación superior en el mundo.

La estadística básica de la DGEST indica que la matrícula oficial del ciclo escolar 2012-2013 fue de 465,483 estudiantes de licenciatura, de los cuales el $61.5 \%$ está en los Institutos Tecnológicos Federales y el 38.5\% en los Institutos Tecnológicos descentralizados. Representa el 14.2\% de la matrícula atendida en educación superior pública en México (Sistema Nacional de Educación Superior Tecnológica, 2012). 


\section{El Instituto Tecnológico de Mexicali}

El ITM se encuentra ubicado en Mexicali, Baja California, México. Inició actividades el 19 de octubre de 1981 (Castro Bojórquez, 2006). Se encuentra Certificado en ISO 9001:2008/NMX-CC-9001-IMNC-2008. Actualmente cuenta con 11 carreras de Ingeniería que son: Sistemas Computacionales, Informática, Industrial, Mecánica, Mecatrónica, Química, Eléctrica, Electrónica, en Energías Renovables, en Gestión Empresarial y en Logística (ITMexicali, 2012).

El ITM, está organizado de manera departamental, igual que todos los tecnológicos del SNIT. Cada carrera es coordinada por un jefe de departamento, que a su vez conforman la Subdirección Académica. Existen otras dos subdirecciones, de Planeación y Administrativa, que no tienen injerencia directa con los departamentos académicos, pero sus actividades están encaminadas al apoyo del trabajo académico, tanto de estudiantes como del personal docente. Se trabaja con un modelo curricular semiflexible, de tal manera que los estudiantes elijen que asignaturas cursar, elijen horarios y profesores. Hay un coordinador por cada carrera de ingeniería, que autoriza la carga académica semestral de los estudiantes.

La distribución de estudiantes de ingeniería inscritos en 2013-1 se presenta en la Tabla 1. La matrícula total de estudiantes fue de 3,108 (en carreras de ingeniería, contador público, informática, contaduría y posgrado), de los cuales el $90.6 \%$ $(2,817)$ cursa carreras de ingeniería.

Tabla 1. Población estudiantil de ingeniería del ITM al 2013-1.

\begin{tabular}{lc}
\hline Carrera & Total \\
\hline Ing. Sistemas Computacionales & 343 \\
Ing. Industrial & 692 \\
Ing. Mecánica & 298 \\
Ing. Química & 216 \\
Ing. Eléctrica & 191 \\
Ing. Electrónica & 146 \\
Ing. en Energías Renovables & 89 \\
Ing. Mecatrónica & 571 \\
Ing. en Logística & 73 \\
Ing. en Gestión Empresarial & 141 \\
Ing. en Informática & 57 \\
\hline Total & $\mathbf{2 . 8 1 7}$ \\
\hline
\end{tabular}

Fuente: Centro de Cómputo del ITM. 


\section{Metodología}

El estudio utilizó como técnica el diseño y aplicación de una encuesta para recabar información sobre las causas de la reprobación y conocer cómo conciben el problema los participantes y cómo se comportan ante él.

Para el estudio se utiliza una metodología descriptiva analítica.

El total de académicos durante el semestre 2013-1 fue de 153; mientras que el número de los administradores o jefes de departamento académico y subdirectores y director llegó a 10. El muestreo realizado fue aleatorio simple, la encuesta se aplicó en febrero de 2013 a la población de académicos y administradores del ITM. Finalmente se recabaron 73 encuestas de profesores y 7 de los administradores.

Se diseñaron y aplicaron dos encuestas para recabar la información:

a) Encuesta para académicos. Se conforma con 12 preguntas abiertas como: antigüedad, departamento de adscripción, sexo, tipo de nombramiento (de tiempo completo o asignatura), carga académica por semestre (número de materias/ horas de clase a la semana), materias que imparte, el porcentaje de reprobación y la carrera, así como preguntas para conocer qué actividades realiza el docente cuando sus estudiantes reprueban. También contiene 4 preguntas dónde el docente selecciona la respuesta, si los estudiantes asisten a asesorías, cómo están las materias que desea llevar y preguntas relativas al servicio del centro de información y de orientación educativa.

b) Encuesta para administradores (jefes de departamento académicos, jefe del Departamento de Ciencias básicas, Subdirector Académico, Subdirector de Planeación y Director de la institución). Este cuestionario contiene 12 preguntas abiertas como: puesto administrativo, antigüedad en este puesto y cómo docente, sexo, materias en las que se presenta el mayor porcentaje de reprobación, cómo es la carga académica de los docentes a su cargo, estrategias y acciones departamental implementadas para disminuir los porcentajes de reprobación así como su impacto y preguntas relativas al servicio del centro de información y de orientación educativa.

Para interpretar el punto de vista de académicos y administradores sobre las causas de reprobación, en los dos instrumentos, se les pregunta el grado de influencia que tienen 29 factores. Las respuestas están basadas en una escala de Likert de cuatro opciones Nada, Poca, Mediana y Mayor.

La validación de las encuestas se realizó mediante la aplicación de la prueba Alpha de Cronbach, la cual se analizó con el paquete estadístico SPSS y se obtuvo un valor de 0.83 , lo que da validez a la encuesta. 
Se encuestaron académicos de los departamentos de: Ciencias Básicas, MetalMecánica, Industrial, Química, Sistemas Computacionales y Eléctrica-Electrónica, quienes atienden a los estudiantes de las 11 carreras de ingeniería que ofrece la institución. Se analizó el $47.7 \%$ de las encuestas a docentes y se encuestó al 70\% de los administradores.

Para asignar un orden de prioridades a las causas de reprobación, se usó el diagrama de Pareto. Mediante el principio de Pareto, se pueden detectar las causas que tienen más relevancia. Se afirma que tanto la distribución de los efectos como sus posibles causas no es un proceso lineal, sino que el $20 \%$ de las causas totales hace que sean originados el $80 \%$ de los efectos (Gutiérrez Pulido y De la Vara Salazar, 2004).

\section{Resultados y discusión}

Las causas de reprobación presentadas en las dos encuestas se clasificaron considerando su origen en: psicológicas, sociales y familiares, atribuible al rendimiento escolar (Espinoza García, 2005), tomando en cuenta que pueden ser atribuibles a la institución, atribuibles al profesor o bien, físicas y económicas. En la Tabla 2 se contempla esta subclasificación.

Tabla 2. Clasificación de las causas de reprobación según su origen.

\section{Causas de reprobación Causas de reprobación
según su origen}

\begin{tabular}{|c|c|}
\hline Atribuibles a la institución & $\begin{array}{l}\text { Muchos estudiantes por grupo, programas extensos, } \\
\text { profesores que no domina el tema o asignatura, } \\
\text { laboratorios y talleres con deficiencia. }\end{array}$ \\
\hline Atribuibles al maestro & $\begin{array}{l}\text { El profesor no explica con claridad los temas, el maestro es } \\
\text { muy exigente, insuficiente material didáctico, los exámenes no } \\
\text { evalúan los temas tratados en clase. }\end{array}$ \\
\hline $\begin{array}{l}\text { Atribuibles al rendimiento } \\
\text { escolar }\end{array}$ & $\begin{array}{l}\text { Falta de motivación para el estudio, no resuelven ejercicios } \\
\text { adicionales, falta de hábitos de estudio, falta de bases } \\
\text { para cursar la materia, tienen dificultad para entender } \\
\text { las materias, no prestan atención a las explicaciones, no } \\
\text { asisten a asesorías y no toman notas ni apuntes en clase. }\end{array}$ \\
\hline
\end{tabular}

Físicas y económicas Falta de recursos económicos y problemas físicos.

Falta de tiempo, no les gusta la materia, su trabajo no les

Psicológicas permite estudiar, problemas emocionales, no les gusta la carrera y no desean seguir estudiando.

No concluyen la materia, faltan mucho a clases, problemas

Sociales y familiares familiares, hijos(as) pequeños(as) que cuidar y embarazo (propio o de su pareja). 


\section{Causas de reprobación desde la perspectiva del profesor}

En la Tabla 3, se presenta el total de profesores por departamento y la muestra de encuestados en el 2013-1. Se observa que fueron los del Departamento de Ciencias Básicas y Química, los que colaboraron más al contestar la encuesta. El jefe del Departamento de Ciencias Básicas, reporta en la encuesta, que los mayores problemas de reprobación se presentan en asignaturas como Física, Cálculo, Cálculo Integral, Química, Química Inorgánica, que son las que enseñan los profesores de este departamento. Los profesores de los Departamentos de Ingeniería Industrial y Sistemas Computacionales fueron quienes mostraron menor disposición para contestar la encuesta. En general, se encuestó el 47.7\% de los académicos.

Tabla 3. Número de profesores por departamento y encuestados en el 2013-1.

\begin{tabular}{lccc}
\hline Profesores por departamento & Total & Encuestados & \% encuestados \\
\hline Sistemas Computacionales & 29 & 10 & 34.5 \\
Industrial & 36 & 11 & 30.6 \\
Metal-Mecánica & 22 & 8 & 36.4 \\
Química & 14 & 11 & 78.6 \\
Ciencias Básicas & 26 & 22 & 84.6 \\
Eléctrica-Electrónica & 26 & 11 & 42.3 \\
\hline Total & $\mathbf{1 5 3}$ & $\mathbf{7 3}$ & $\mathbf{4 7 . 7}$ \\
\hline
\end{tabular}

Fuente: Depto. de Recursos Humanos e información de la encuesta causas de reprobación. ITM 2013.

En la Figura 1, se presentan las causas de reprobación agrupadas según la subclasificación de Espinoza García (2005). El 80-20 de las causas de reprobación desde la perspectiva del académico se debe a: causas atribuibles al rendimiento escolar, causas psicológicas, causas sociales y familiares y causas atribuibles a la institución. Es decir desde la perspectiva del académico el estudiante reprueba principalmente por problemas tales como falta de bases para cursar la materia, de hábitos de estudio, no prestar atención, no asistir a asesorías y falta de motivación para el estudio. 
Figura 1. Causas de reprobación desde la perspectiva del académico $(\mathrm{N}=73)$. Fuente: Encuesta causas de reprobación. ITM 2013.

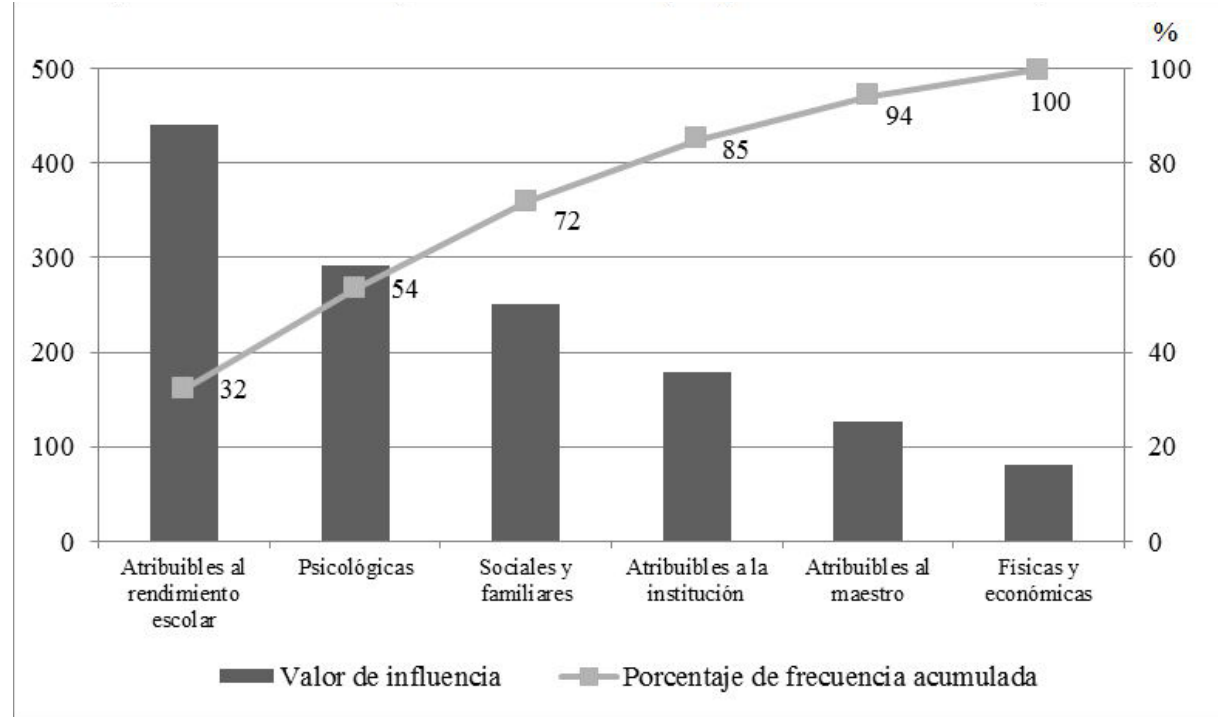

Fuente: Encuesta causas de reprobación. ITM 2013.

El diagrama de Pareto de la Figura 1 indica que el $80 \%$ de las causas que generan la reprobación según el orden de prioridad, mencionadas por los académicos, son: a) atribuibles al rendimiento escolar como las habilidades del estudiante para estudiar, lo cual puede deberse a que están acostumbrados a no tomar apuntes en clase, a dedicar pocas horas al estudio, entre otras cosas; b) causas psicológicas, como son los problemas emocionales, falta de tiempo para el estudio, falta de deseo de continuar con los estudios, otras prioridades sobre el estudio como el trabajo, no encuentran gusto a la materia, estas causas hacen ver que los estudiantes que reprueban, no tienen un plan de vida donde su prioridad sea concluir la carrera, muchos de ellos están estudiando determinada carrera que fue su segunda o tercer opción y tal vez no quedaron en la IES que deseaban, esto los ha llevado a reprobar materias y al no ser regulares no han podido cambiar de carrera, resignándose de alguna manera a esta; c) otras causas importantes en el 80-20 son las causas sociales y familiares, en estas se contemplan problemas como embarazos, cuidados de hijos menores, así como problemas familiares, estos problemas pueden deberse a que muchos de los estudiantes del ITM pertenecen a estrato económico de medio a bajo, y por ello se ven obligados a trabajar para mantener a su familia reduciendo así el tiempo que le dedican al estudio de sus materias; d) y las causas atribuibles a la institución, como grupos muy numerosos que dificultan al académico proporcionar la atención debida a cada estudiante. 


\section{Causas de reprobación desde la perspectiva los administradores}

En la Figura 2, se muestra el Pareto de causas posibles de la reprobación de los estudiantes, según el orden de prioridad de los administradores. El 80-20 corresponde a las causas atribuibles: al rendimiento escolar, al maestro, a la institución y a causas psicológicas.

Figura 2. Causas de reprobación desde la perspectiva de los administradores $(\mathrm{N}=7)$.

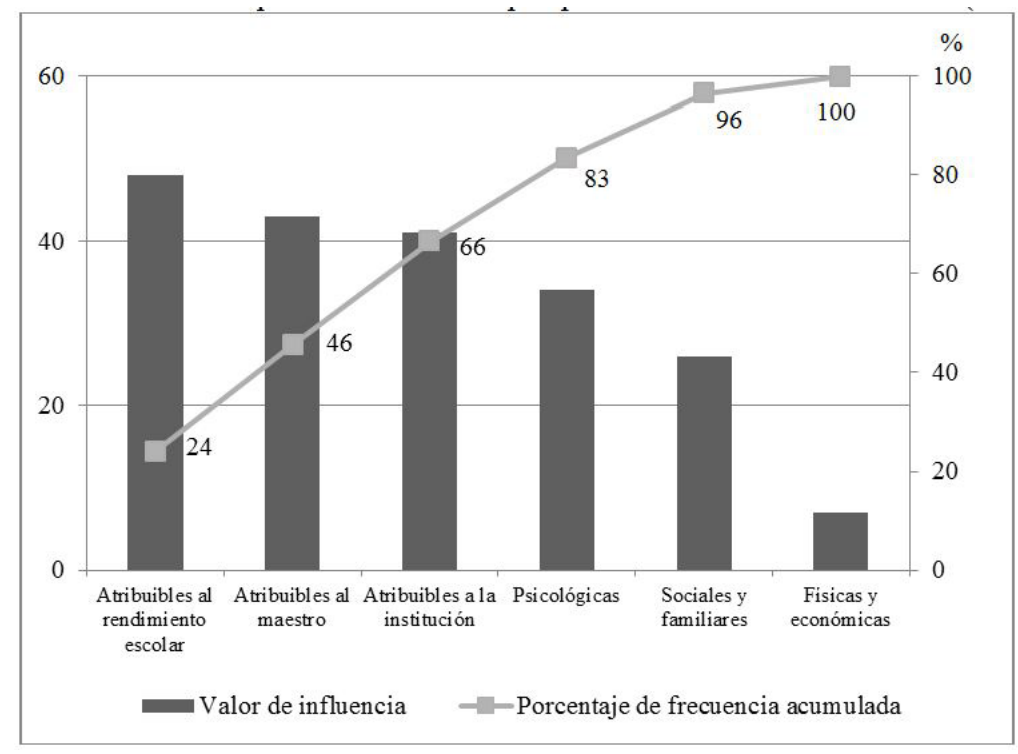

Fuente: Encuesta causas de reprobación. ITM 2013.

El 80-20 de las causas de reprobación desde la perspectiva de los administradores se debe: a) causas atribuibles al rendimiento escolar, b) causas atribuibles al maestro, c) causas atribuibles a la institución y d) causas psicológicas. Es decir problemas relacionados a la falta de hábitos de estudio, de bases para cursar la materia, dificultad para entender la materia, no tomar notas ni apuntes, así como no asistir a asesorías. Los administradores del ITM están conscientes que, los estudiantes que han reprobado al menos una materia, presentan carencias y debilidades para el estudio, situación que puede deberse a la falta de un buen proceso de selección de estudiantes de nuevo ingreso, y que se agrava al considerar que el Instituto Tecnológico de Mexicali no es la primera opción para los estudiantes que desean estudiar una carrera en la ciudad de Mexicali, Baja California, México.

Así mismo, los administradores consideran que la segunda causa en importancia para la reprobación de los estudiantes es la atribuible al maestro. Problemas tales 
como: el profesor no explica con claridad, no proporciona el suficiente material didáctico, exámenes que no evalúan los temas vistos y maestros exigentes. Es importante resaltar que los administradores están conscientes que el fenómeno de la alta reprobación se debe, en gran medida, a las ineficiencias del profesorado. Por ello es interesante conocer las acciones que los administradores están tomando para reducir esta causa en cada uno de sus departamentos. Cabe mencionar que dos veces por año se imparten cursos de formación y actualización docente organizados por el Departamento de Desarrollo Académico. Lo reportado por los estudiantes sugiere que quizás estos cursos no están enfocados a reducir las causas que originan la reprobación (Amado et al, 2013). Por otro lado, es importante analizar también los mecanismos de incorporación de los académicos al ITM.

El administrador también está consciente que causas atribuibles a la institución, ocupan un lugar importante en la reprobación de los estudiantes. Estas contemplan problemas como: muchos estudiantes por grupo, profesores que no dominan el tema, laboratorios y talleres con deficiencias y programas extensos.

Así mismo, el administrador considera que causas psicológicas como: problemas emocionales de los estudiantes, falta de deseo de seguir estudiando, falta de gusto por la carrera y falta de tiempo son causas que contribuyen de manera importante en la reprobación.

En las respuestas a las preguntas abiertas del cuestionario los jefes académicos y/o subdirector mencionan, de acuerdo a su experiencia, que las asignaturas en las que se presentan el mayor porcentaje de reprobados son en los primeros semestres: Física (Electromagnetismo, Estática y Dinámica), Química Inorgánica, todas las Matemáticas, Métodos Numéricos, Programación en todas las carreras de ingeniería, Fundamentos de Programación, Estructura de Datos, Sistemas Operativos, Matemáticas, Ingeniería Económica.

Los administradores afirman que la asignación de las materias es con base en el perfil de los profesores y su experiencia profesional. En tanto que, la carga académica de los docentes está programada con base al reglamento, es decir, de acuerdo a su nombramiento, sean de tiempo completo, $3 / 4$ de tiempo, 1/2 tiempo o de asignatura. Lo anterior es la razón por la que varían las horas de descarga para llevar a cabo actividades inherentes a la docencia como asesorías o asistencia a cursos, con lo que mejorarían su desempeño.

Han implementado estrategias departamentales para disminuir los porcentajes de reprobación como: cursos remediales, asesorías con docentes y estudiante-estudiante, curso Propedéutico de Matemáticas (Álgebra) a estudiantes de nuevo ingreso y cuentan con un Programa Institucional de Tutorías (de la DGEST). Sin embargo, consideran que el impacto de las estrategias implementadas es bajo y lento, aunque el Jefe del Departamento Ciencias Básicas reportó en la encuesta, que el curso Propedéutico de Matemáticas ha disminuido la reprobación en Cálculo Diferencial. 
Consideran, los administradores, que los estudiantes reprueban porque: no están convencidos de la carrera que estudian, tienen malos hábitos de estudio, les falta vocación, tienen problemas de actitud, no valoran la carrera, les faltan bases de aritmética, un buen porcentaje de estudiantes falla en las tablas de multiplicar y en operaciones con fracciones, no muestran mucho compromiso de su parte. También la actitud de los docentes hacia los estudiantes puede estar desmotivada por lo que difieren en intereses, además no se ha considerado que las nuevas generaciones presenten una dinámica muy diferente de las generaciones pasadas, que las técnicas de enseñanza actualmente utilizadas por los docentes pudieran ser obsoletas, aunado a maestros que requieren motivación y entusiasmo.

Las acciones que proponen los administradores y están dispuestos a medir, analizar, controlar e implementar para disminuir la reprobación son:

- Seguimiento puntual al contenido de las asignaturas que se trabaja en el grupo, con implementación de medidas aplicables para todos los académicos, sin preferencias. - Sobre la evaluación mencionan que: hay que supervisar la aplicación del Modelo educativo basado en competencias, para priorizar el "cómo lo hacen" y el "cómo lo utilizan", evitando que sea considerado como único criterio para evaluar, los exámenes.

- Medir la cantidad de estudiantes que no aprueban las materias del área de eléctrica-electrónica.

- Implementar cursos propedéuticos en las disciplinas que lo requieran.

- Analizar y realizar acciones para disminuir la reprobación con base en los datos que proporciona el Sistema Integral de Información (SII).

- Implementar un grupo académico de seguimiento a la problemática de la reprobación, algo parecido a las reuniones de calidad que realiza la Dirección de la Institución.

\section{A manera de conclusiones}

El análisis realizado no permite afirmar que la deficiencia en conocimientos, la planeación académica, la práctica docente, la situación económica, son causas significativas de reprobación; tampoco lo es la falta de interés del estudiante por el estudio, ni la situación económica.

Cada uno de los actores que contestaron la encuesta de causas de reprobación considera como de mayor influencia las siguientes:

Por su lado, los estudiantes consideran que las causas atribuibles al rendimiento escolar (falta de motivación para el estudio), atribuibles al maestro (el profesor no explica con claridad los temas) y a la institución (programas extensos), son las de mayor influencia en la reprobación (Amado et al, 2013). 
En este documento se muestra que los profesores consideran, en general, que las causas atribuibles al rendimiento escolar (falta de hábitos para el estudio), causas psicológicas (falta de tiempo para estudiar), causas sociales y familiares (faltan mucho a clases), y las atribuibles a la institución (muchos estudiantes por grupo) son las de mayor influencia en la reprobación.

Los administradores, al igual que los estudiantes (Amado et al, 2013), consideran en general que las causas atribuibles al rendimiento escolar (no resuelven ejercicios adicionales), atribuibles al maestro (el profesor no explica con claridad los temas), a la institución (muchos estudiantes por grupo) y psicológicas (falta de tiempo para estudiar) son las de mayor influencia en la reprobación.

Los estudiantes proponen acciones para abatir la reprobación como: poder realmente elegir al profesor, que las asignaturas se ofrezcan con distintos profesores y en diferentes horas, horarios más cómodos y maestros con más dedicación a los estudiantes y también grupos más pequeños, para que lo anterior sea posible (Amado et al, 2013).

Mientras, para disminuir la reprobación, los profesores proponen: programas de asesorías de Álgebra y Cálculo Diferencial, para que adquieran los conocimientos previos, cursos simultáneos de Álgebra principalmente, asesorías extra clase, manejo de puntos extras para motivar a los estudiantes, tarea por equipo, no permitir a los coordinadores de carrera asignar asignaturas seriadas sin haber llevado la anterior, más trabajo en el aula, con clases interactivas que privilegien la multimedia, hacerles conciencia de lo importante que es su carrera, trabajo de academia para el personal docente, promover círculos de estudio eficientes, sensibilizar al estudiante sobre la importancia de estudiar, tener grupos no mayores de treinta estudiantes, curso obligatorio de Álgebra y no inscribirlos en un curso sin haber aprobado el anterior, mayor capacitación docente sobre el comportamiento del estudiante, que en tutorías se revisen hábitos de estudio, hacer un buen examen de selección, entre otras.

A su vez los administradores proponen para disminuir la reprobación: seguimiento puntual al contenido que se da frente a grupo con implementación de medidas aplicables para todos sin preferencias, supervisar la aplicación del Modelo educativo basado en competencias para priorizar el "cómo lo hacen", y el "cómo lo utilizan" y evitar la aprobación de exámenes como el único criterio para evaluar. De igual manera se propone medir la cantidad de estudiantes que no aprueban las materias del área de eléctricaelectrónica, implementar cursos propedéuticos, que los dejen analizar y realizar acciones para disminuir la reprobación con datos proporcionados por el Sistema Integral de Información e implementar un grupo de profesores que dé seguimiento a este problema, algo parecido a las reuniones de calidad que realiza la Dirección de la institución.

Como se observa las causas son muchas y dependiendo de la perspectiva de los actores educativos, unas serán más significativas que otras. El fenómeno es complejo, sin embargo ya está el punto de partida, pues los actores que influyen en esta problemática, quieren erradicarla y deben implementar acciones para lograrlo. 


\section{Algunas propuestas}

El fenómeno de la reprobación en las instituciones de educación superior ocasiona la incorporación tardía de los jóvenes al campo laboral y profesional, algunos son orillados a la deserción escolar y es también, una pérdida de recursos económicos para la institución y el país.

Con base en el resultado del análisis realizado, se formulan las siguientes propuestas:

- Diseñar e impartir semestralmente cursos-talleres de hábitos de estudio, métodos y estrategias de enseñanza-aprendizaje, redacción y comprensión lectora.

- Capacitar a los docentes para detectar problemas que afecten al rendimiento escolar, psicológico y de salud de los estudiantes.

- Crear programas efectivos para detectar a estudiantes con problemas académicos, emocionales y psicológicos.

- Proporcionar, en la institución, un área específica de atención psicológica al estudiante, cuestión desatendida en el instituto. 


\section{Referencias}

Amado Moreno, M. G., García Velázquez, A., Sánchez Luján, B. I., Brito Páez, R. A. (2013). Causas de reprobación en ingeniería, visión del estudiante. V Congreso Internacional de Educación. Universidad Autónoma de Baja California, 13-16 nov., Mexicali, Baja California, México.

Castro Bojórquez, R. (2006). Hacia un nuevo Tecnológico 25 años, 1981-2006. México: Instituto Tecnológico de Mexicali.

Durán Encalada, J.A., Díaz Hernández, G. (1990). Análisis de la deserción estudiantil en la Universidad Autónoma Metropolitana. Revista de la Educación Superior, Volumen 19 (74). Recuperado de http://publicaciones.anuies.mx/revista/74.

Espinoza García, C. et al. (2005). Propuesta de sistema integral de tutorías académicas para el nivel medio superior universitario. Foro Reforma del Bachillerato Universitario. Benemérita Universidad Autónoma de Puebla, 28 30 nov., Puebla, Puebla, México.

González Montes, A., Rodríguez Galván, L. F., Treviso Burciaga, J. L., Flores Payán, S., Makita Aguilar, M. A. (2011). Factores que afectan el rendimiento escolar de los alumnos de ingeniería industrial del Instituto Tecnológico de Chihuahua II. $4^{\circ}$ Congreso Internacional de Investigación. 27-29 sept., Nuevo Casas Grandes, Chihuahua, México.

Gutiérrez Pulido, H., De la Vara Salazar, R. (2004). Control Estadístico de Calidad y Seis Sigma. México: Editorial McGrawHill.

ITMexicali, (2012). Recuperado de www.itmexicali.edu.mx.

Mánica Zuccolotto, C., Cetina Canto, T. E., Pinzón Lizárraga, L. M. (2008). Propuestas para disminuir el índice de reprobación en una IES bajo una perspectiva de Desarrollo Organizacional. Octavo foro de Evaluación Educativa. 22 - 24 oct., Mérida, Yucatán, México.

Morales Burgos, A., García Sosa, J., Escalante Triay, E. (2009). Causas de reprobación en los cursos de Mecánica de Fluidos de la Facultad de Ingeniería de la Universidad Autónoma de Yucatán. Ingeniería Revista Académica de la FI-UADY, Volumen 13 (3), pp 45-51.

Nava Bustos, G., Rodríguez Roldán P., Zambrano Guzmán, R. (2007). Factores de reprobación en los alumnos del Centro Universitario de Ciencias de la Salud de la Universidad de Guadalajara. Revista de educación y desarrollo. Volumen 7 , pp $17-25$. 
Sistema Nacional de Educación Tecnológica, (2012), Anuario estadístico 2012, México, SEP-SNEST-DGET. Recuperado de www.dgest.gob.mx. [Consulta: mayo de 2013].

Vega Pérez, L. G. (Coordinador) (2012). Modelo Educativo para el Siglo XXI: formación y desarrollo de competencias profesionales, México: Secretaría de Educación Pública, Dirección General de Educación Superior Tecnológica.

\section{Agradecimientos}

Este proyecto no hubiese sido posible sin la colaboración de estudiantes, académicos, jefes de departamento, subdirectores y estudiantes prestadores de servicio social. A todos ellos muchas gracias. 
